\title{
$1495-1497$
}

\author{
FRANK ZÖLLNER
}

\section{LEONARDO DA VINCIS »ABENDMAHL«. ZWISCHEN KANONISIERUNG UND KONTEXTUALISIERUNG}

Wenn vom Kanon der Kunstgeschichte die Rede ist, dann führt kein Weg an den Werken Leonardo da Vincis vorbei. Mindestens zwei seiner Gemälde behaupten seit Jahrhunderten Spitzenpositionen in der kunsthistorischen Bewertungsskala, das zwischen 1495 und 1497 für Ludovico Sforza entstandene »Abendmahl« im Refektorium des Mailänder Klosters S. Maria delle Grazie (Abb. 1-2) ${ }^{1}$ und das 1503 von dem Florentiner Seidenhändler Francesco del Giocondo in Auftrag gegebene Bildnis seiner Gattin Lisa del Giocondo, die so genannte "Mona Lisa« im Pariser Louvre. ${ }^{2}$ An der Rezeptionsgeschichte dieser beiden Gemälde lassen sich die wechselhaften Prozesse der Kanonbildung exemplarisch verfolgen. Stand zunächst das "Abendmahl « ganz oben auf der Liste der bekanntesten Renaissancegemälde, so hat im 20. Jahr-
1 Die folgenden Ausführungen fußen auf mehreren bereits publizierten Vorarbeiten, besonders auf Frank Zöllner, Leonardo da Vinci. Sämtliche Gemälde und Zeichnungen, 3. Aufl., 2 Bde., Köln u. a. 2011, Bd. 1, 122-139, 206-207, 230-232; für weiterführende Angaben verweise ich auf Alessandro Nova, Sabine Feser (Hrsg.), Giorgio Vasari, Das Leben des Leonardo da Vinci, übers. v. Victoria Lorini, Berlin 2006, 28-32, 81-84. Grundlegend zum »Abendmahl«: Emil Möller, Das Abendmahl des Lionardo da Vinci, Baden-Baden 1952; Ludwig H. Heydenreich, Invito a Leonardo. L'ultima cena, Mailand 1982 (zuerst engl. 1974); Pinin Brambilla Barcilon, Pietro C. Marani, Leonardo. L'ultima cena, Mailand 1999; Leo Steinberg, Leonardo's Incessant Last Supper, New York 2001. Zur grundsätzlichen Problematik des Kanons in der Kunstgeschichte siehe Frank Zöllner, Kanon und Hysterie. Primavera, Mona Lisa und die Sixtina im Chaos der Deutungen, in: Kunstgeschichte. Open Peer Reviewed Journal Juni 2010, URL: http://www.kunstgeschichte-ejournal.net/archiv/2010/zoellner/.

2 Roy McMullen, Mona Lisa. The Picture and the Myth, London 1976; Frank Zöllner, Leonardos Mona Lisa. Vom Portrăt zur Ikone der freien Welt, Berlin 2006; Ders., (wie Anm. 1), 5, 154-161, 208, 240-241, 251-252; Donald Sassoon, Leonardo and the Mona Lisa Story. The History of a Painting Told in Pictures, London 2006. 
ABB. 1: LEONARDO DA VINCI, DAS ABENDMAHL, CA. 1495-1497, TEMPERA AUF PUTZ, $460 \times 880$ CM, MAILAND, SANTA MARIA DELLE GRAZIE, NORDWAND DES REFEKTORIUMS

hundert die »Mona Lisa" dessen Platz eingenommen und bis heute behalten. ${ }^{3}$

Die Ablösung des »Abendmahls« als absolutes Spitzenstück des Kanons durch die Mona Lisa zeichnete sich bereits im 19. Jahrhundert ab. Das »Abendmahl" hatte zunächst durch seine Bindung an einen für Teile des Publikums zugänglichen Raum eine breitere Wirkung als die »Mona Lisa«. Das änderte sich zu Beginn des 19. Jahrhunderts mit der Eröffnung des Pariser Louvre als öffentlicher Kunstsammlung. Die Mona Lisa wurde quasi wieder entdeckt, wobei sie gegenüber dem »Abendmahl« einen entscheidenden Vorteil besaß: Man konnte sie als ikonisches Einzelbild wahrnehmen, das weitgehend frei von Attributen und konkreten Zeitbezügen jedem historischen Zusammenhang enthoben schien. Damit war das Bildnis im Louvre als Projektionsfläche für die Phantasien der Betrachter geeigneter als das "Abendmahl", dessen Ikonographie und Kontext der Deutungswut gewisse Grenzen setzten. Zudem zementierten mehrere medienwirksame Ereignisse des 20. Jahrhunderts die Spitzenposition der "Mona Lisa« im Kanon der Kunstgeschichte: der spektakuläre Raub des Gemäldes im August 1911, seine ebenso spektakuläre Rückführung in den Louvre im Dezember 1913 und seine Aufsehen erre-

3 Hans Belting, Das unsichtbare Meisterwerk. Die modernen Mythen der Kunst, München 1998, 63-82, 167-186, 310-332; vgl. auch George Boas, The Mona Lisa in the History of Taste, in: Journal of the History of Ideas, 1, 1940, 207-224; Andre Chastel, L'Illustre incomprise. Mona Lisa, Paris 1988; Isa Bickmann, Leonardismus und symbolistische Ästhetik. Ein Beitrag zur Wirkungsgeschichte Leonardo da Vincis in Paris und Brüssel, Frankfurt/M. 1999. 


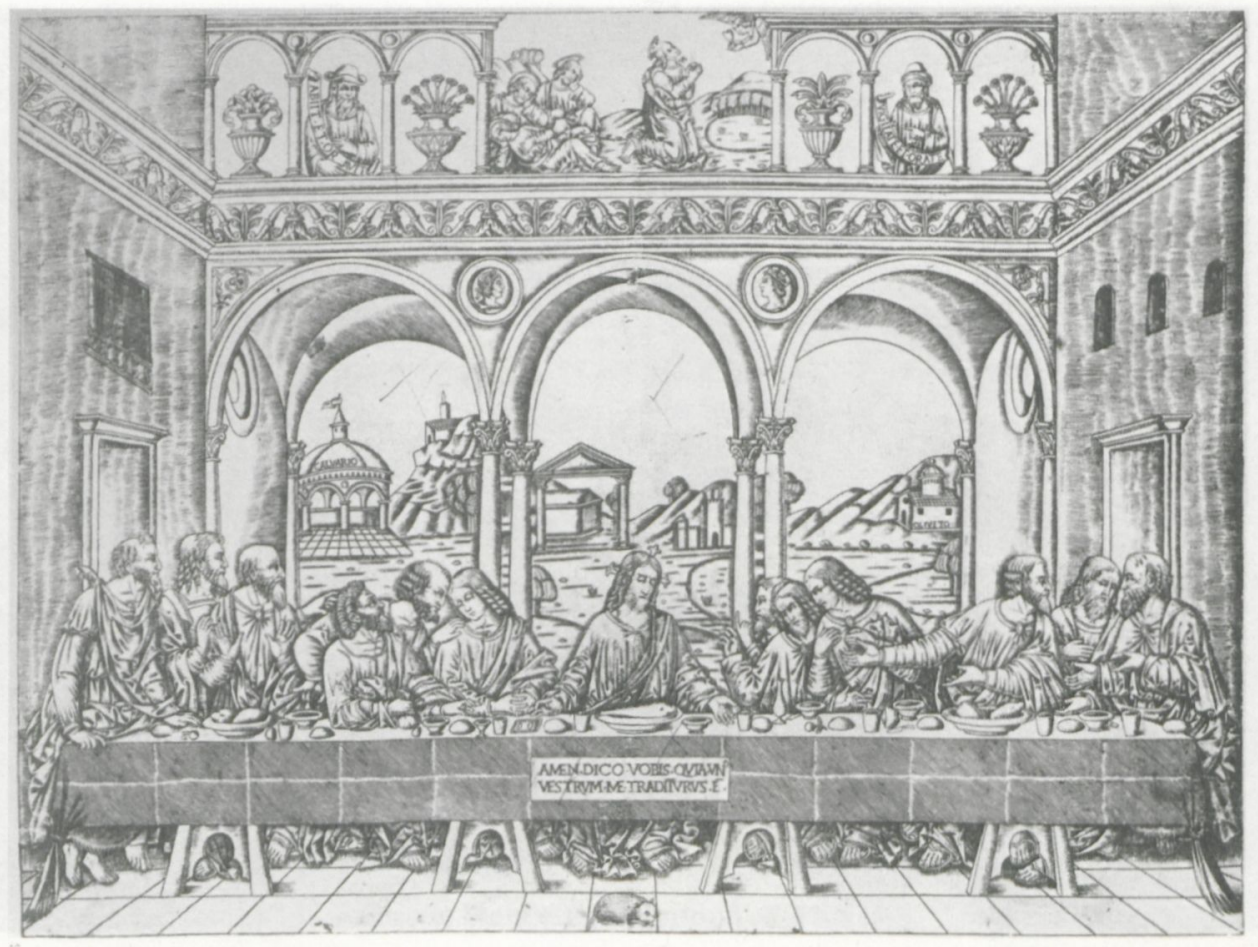

ABB. 3: UNBEKANNTER KONSTLER, KOPIE NACH LEONARDOS ABENDMAHLs, CA. 1498,

KUPFERSTICH, $322 \times 425$ MM, PRIVATSAMMLUNG

dem das "Abendmahl« seinen Platz an der Spitze des Kanons längst verloren hat, ist Leonardos Wandbild die mit Abstand bekannteste Variante dieses Bildthemas.

Ebenso wie die zahlreichen Reproduktionen bezeugen auch die frühesten Schriftquellen eine außerordentlich hohe Wertschätzung des Bildes. Zu den ersten Kommentatoren gehört der Mathematiker Luca Pacioli, ein Freund Leonardos, der das Werden des Wandgemäldes in Mailand unmittelbar erlebt hatte und es sofort nach dessen Fertigstellung begeistert beschrieb. Er lobt vor allem die Lebensechtheit der Darstellung: Es sei

wnicht möglich, sich die Apostel lebendiger vorzustellen beim Klang der Stimme der untrüglichen Wahrheit, als Jesus sprach: 'Einer von euch wird mich verraten, wo in Haltung und Gestik der eine zum anderen und dieser zu jenem mit lebhaftem und traurigem Erstaunen zu reden scheint, so würdig ordnete es unser Leonardo mit seiner gewandten Hand an. $\kappa^{5}$
5 Luca Pacioli, De Divina Proportione, Venedig 1509, c. 3, zit. nach Edoardo Villata, Leonardo da Vinci. I documenti e le testimonianze contemporanee, Mailand 1999, $\$$ 124b; dt. Öbers. nach Luca Pacioli, Divina proportione. Die Lehre vom Goldenen Schnitt [1509], hrsg. u. übers. v. Constantin Winterberg, Wien 1889, 41 [entstanden 1497/1498]. Die Quellen zum »Abendmahle in der Originalsprache finden sich bei Zöllner (wie Anm. 1), 207-208; vgl. auch Leonardo da Vinci. Eine Biographie in Zeugnissen, Selbstzeugnissen, Dokumenten und Bildern, hrsg. u. komm. v. Marianne Schneider, München 2002 , 117-126. 
Nicht weniger angetan war der Reisebegleiter des Kardinals d'Aragon, Antonio de Beatis, der im Dezember 1517 das Refektorium der Mailänder Dominikaner besuchte und Leonardos Gemälde folgendermaßen kommentierte:

"Es ist ganz ausgezeichnet, obwohl es bereits beginnt, schadhaft zu werden - ich weiß nicht, ob wegen der Feuchtigkeit, die dem Mauerwerk anhaftet, oder wegen einer anderen Unachtsamkeit. Die Personen jenes Abendmahls sind nach der Natur gemalte Bildnisse von mehreren Personen am Hofe und von Mailändern jener Zeit in wahrer Lebensgröße. Dort sieht man auch eine Sakristei, sehr reich an Brokatparamenten, gleichfalls gestiftet von dem genannten $\mathrm{Lu}$ dovico ${ }^{6}{ }^{6}$

I.

\section{IKONOGRAPHIE UND DARSTELLUNG DES BILDSUJETS}

Bereits mit den frühesten Beschreibungen sind einige der wichtigsten Themen vorgegeben, die in den folgenden Jahrhunderten die Debatte um das "Abendmahl" bestimmen sollten: der beklagenswerte Verfall des in einer prekären Maltechnik ausgeführten Werks sowie die lebensechte Darstellung einer Vielfalt gestischer und mimischer Ausdruckswerte. Diese Vielfalt hängt unmittelbar mit Leonardos besonderer Auslegung des Bildsujets zusammen, wenn er in seinem Gemälde vor allem die Verratsankündigung darstellt. Tatsächlich konzentriert sich der Künstler mit seiner Darstellung auf eben jenen Moment, in dem Jesus mit seinen Jüngern zu Tisch sitzt und verkündet: »Wahrlich, ich sage euch, einer unter euch wird mich verraten « (»Amen dico vobis, quia unus vestrum me traditurus est «, Mt. 26.21). Mit den verschiedensten Gesten und Reaktionen bringen daraufhin fast alle Jünger ihr Erstaunen und Erschrecken über die Ankündigung des Verrats zum Ausdruck: Am linken Ende der Tafel erhebt sich Bartholomäus aufgebracht von seinem Stuhl, neben ihm reißen Jacobus minor und Andreas erstaunt die Hände in die Höhe. Petrus erhebt sich ebenfalls von seinem Sitz und wendet sich mit zornigem Gesicht der Bildmitte zu. Vor ihm erscheint der Verräter Judas, der sich erschrocken zurücklehnt und mit seiner rechten Hand den Geld-
6 Ludwig v. Pastor, Die Reise des Kardinals Luigi d'Aragona durch Deutschland, die Niederlande, Frankreich und Oberitalien, 1517-1518, beschrieben von Antonio de Beatis, Freiburg 1905, 176; Villata (wie Anm. 5), $\$ 314$ (unvollständig). Vgl. zum Verfall auch Giorgio Vasari, Le vite dec più eccellenti pittori scultori ed architettori [1568], hrsg. v. Gaetano Milanesi, 9 Bde., Florenz 1906, Bd. 6, 491, der das »Abendmahl 1566 besichtigte; Vasaris Beschreibung des Bildes in der Vita Leonardos ebd., Bd. 4, 29-32. 
beutel mit dem Lohn für seinen Verrat umfasst. Erstmals in der Geschichte der neuzeitlichen Abendmahlsdarstellungen sitzt Judas nicht mehr isoliert vor, sondern hinter dem Tisch. Er taucht 7 Vgl. hierzu Frank Zöllner, Bewegung und Ausdruck bei Leonardo da Vinci, dadurch unmittelbar neben Johannes auf, der eher verhalten reagiert und mit gefalteten Händen fast kontemplativ vor sich hinblickt. Vergleichsweise regungslos folgt an zentraler Stelle, in der Bildmitte vor einer Fensteröffnung postiert, Jesus selbst. Er wird auf der anderen Seite von zwei weiteren Gruppen mit je drei Jüngern flankiert: Thomas, Jacobus major und Philippus sowie Matthäus, Taddeus und Simon.

Die Verratsankündigung führt, wie angesichts der schockierenden Mitteilung Christi nicht anders zu erwarten, zwangsläufig zu einem gewissen Tumult unter seinen Jüngern. Daher dynamisiert Leonardo das Geschehen sowohl durch die Verteilung der zwölf Jünger auf vier unterschiedlich gestaltete Figurengruppen als auch durch die genau kalkulierte Wiedergabe von Gestik und Mimik der einzelnen Personen. Diese außerordentliche Dynamisierung, die Abendmahlsdarstellungen bis dahin fehlte, war im Grunde ein ideales Thema für Leonardo, denn sein Interesse galt besonders der Vielfalt bewegter und ausdrucksstarker Figuren. ${ }^{7}$ So belegen Aufzeichnungen, Ideenskizzen und Vorzeichnungen zum »Abendmahl«, dass der Künstler sehr viel Mühe darauf verwendete, eine große Vielfalt expressiver Gestik und Mimik zu erzielen (s.u.). Dieser Aufwand Leonardos war offenbar so ungewöhnlich und zugleich bei seinen Zeitgenossen so bekannt, dass noch Jahrzehnte später der Schriftsteller Giovanbattista Giraldi davon berichtet:

„Wenn immer Leonardo eine Figur malen wollte, dachte er zuerst über ihre Eigenschaften und ihre Natur nach, d.h. darüber, ob sie edel oder pöbelhaft, heiter oder streng, finster oder froh, alt oder jung, zornig oder ruhigen Gemütes, gut oder böse sein sollte. Und nachdem er ihr Wesen erkannt hatte, ging er dorthin, wo er wusste, dass Personen mit solchen Eigenschaften sich versammelten, und er beobachtete sorgfältig ihre Gesichter, ihr Benehmen, ihre Kleider und Körperbewegungen. Und wenn er etwas gefunden hatte, das für seine Zwecke geeignet erschien, hielt er es mit dem Zei- 
chenstift in einem Büchlein fest, das er stets am Gürtel bei sich trug. Und nachdem er das viele, viele Male getan hatte und so viel zusammengekommen war, wie für das zu malende Bild auszureichen schien, begann er es zu gestalten, und er erreichte, dass es wunderbar gelang. Angenommen, dass er dies in jedem seiner Werke getan hätte, so hat er es auch mit seiner ganzen Sorgfalt in jenem Bild getan, das er in Mailand im Kloster des Predigerordens malte, auf dem unser Erlöser mit seinen Jüngern zu Tisch sitzend abgebildet ist ${ }^{8}{ }^{8}$

$\mathrm{Zu}$ der Schilderung Giraldis finden sich verblüffende Parallelen in Leonardos Aufzeichnungen. So schreibt der Künstler in seinem um 1490 begonnenen und erst im 16. Jahrhundert auszugsweise publizierten Malereitraktat, ein Maler solle sich beim Spazierengehen die Stellungen und Gebärden der Menschen anschauen und in einem Büchlein mit flüchtigen Skizzen festhalten. Die so festgehaltenen Bewegungs- und Ausdrucksmotive würden zu groben Skizzen führen und könnten dann später ausgeschmückt und perfektioniert werden. ${ }^{9}$ In weiteren Aufzeichnungen aus der Mitte der 9oer Jahre des 15. Jahrhunderts finden sich sogar Belege dafür, dass der Künstler eben diese Empfehlungen während seiner vorbereitenden Arbeiten in die Praxis umsetzte. So notiert er in seinem heute als Codex Forster bekannten Notizbuch die Namen mehrerer Personen, deren Hände und Gesichter ihm als Modelle dienen sollten. In demselben Notizbuch schildert er auch die verschiedenen Reaktionen der Jünger auf die Verratsankündigung Christi. Bereits seine vorbereitenden Notizen handeln also vom zentralen Darstellungsgegenstand. ${ }^{10}$

Allerdings setzte Leonardo die im Codex Forster beschriebenen Details nur unvollständig in seinem Gemälde um. So entspricht die Darstellung der Jünger im »Abendmahl« nicht folgender Aufzählung der Personen aus dem genannten Notizbuch: "Einer, der gerade trank, ließ den Becher auf seinem Platz stehen und drehte den Kopf dem Erzählenden zu. Ein Anderer, mit verschränkten Fingern und erhobenen Augenbrauen, wandte sich dem Nachbarn zu «. ${ }^{11}$ Tatsächlich erscheint keine der Figuren exakt in einer ebensolchen Gestalt. Lediglich Johannes, links
8 Giovanbattista Giraldi, Discorsi, Venedig 1554, 193-196; ital. Text bei Giovanni Poggi (Hrsg.), Leonardo da Vinci. La vita di Giorgio Vasari nuovamente commentata, Florenz 1919, 19; dt. Übers. nach Heinz Lüdecke, Leonardo da Vinci im Spiegel seiner Zeit, 2. Aufl., Berlin 1953, 18.

9 Leonardo da Vinci, Das Buch von der Malerei, hrsg. v. Heinrich Ludwig, 3 Bde., Wien 1882, $\S \S 290,173,179$ 189. Vgl. hierzu den klassischen Aufsatz von Ernst H. Gombrich, Leonardo's Method of Working out Compositions, in: Ernst $\mathrm{H}$. Gombrich, Norm and Form, Oxford 1966, 58-63 (zuerst franz. 1954).

10 Jean Paul Richter (Hrsg.),

The Literary Works of Leonardo da Vinci, 2 Bde., 3. Aufl., Oxford 1970, §§ 665-667.

11 Ebd., $\$ 665$. 
von Christus, verschränkt die Finger beider Hände und neigt seinen Kopf der Gruppe von Judas und Petrus zu, doch ohne dabei die Brauen zu heben. Auch die weiteren Beschreibungen ähneln nur teilweise den schließlich im Wandbild realisierten Jüngern. ${ }^{12}$ Diese Unterschiede des ausgeführten Gemäldes zu Leonardos Notizen werfen ein bezeichnendes Licht auf seine Vorgehensweise. Sie bestand einesteils in einer akribischen Vorbereitung in Gestalt zahlreicher Überlegungen und Studien, andernteils aber auch in spontaner Abweichung von der eigenen Planung. Der Vergleich zwischen den zitierten Notizen und dem ausgeführten Bild belegt zudem, dass Leonardo bereits in einer frühen Entwurfsphase daran dachte, den Moment der Verratsankündigung darzustellen.

Leonardos große Sorgfalt bei der Gestaltung des "Abendmahls" wird auch aus seinen Detailstudien zu den Physiognomien der Apostel Jacobus major, Philippus, Judas, Bartholomäus und Petrus deutlich. Hierbei griff er teilweise auf Gesichtstypen zurück, die bereits in der "Anbetung (Anbetung der Könige, 1481/82, Florenz, Uffizien), einem unvollendet gebliebenen Altarbild aus seiner Florentiner Frühzeit, auftauchen: im Falle der Studie für den Apostel Philippus auf einen engelhaften Jüngling und für die Physiognomie des Petrus zunächst auf einen bärtigen alten Mann mit zerfurchtem Gesicht. ${ }^{13}$ Diese Studie fand aber keine Verwendung bei der Ausführung des Gemäldes. Der Künstler benutzte also zumindest im Entwurfsstadium für das »Abendmahl« keine vollkommen neuen Figurentypen. Wohl aufgrund dieses ihm eigenen Defizits strebte Leonardo während der Entstehungszeit nach einer größeren Typenvielfalt, zumal er selbst in seinem Malereitraktat häufig gegen die Wiederholung einander ähnelnder Figuren und Physiognomien polemisiert hatte: Es sei ein sehr großer Fehler der Maler, »dieselben Bewegungen, Gesichter und Gewandstile in ein und derselben Geschichte zu wiederholen und den größten Teil der Gesichter ihrem Meister ähnlich zu machen $[\ldots] \ll .{ }^{14}$
12 Ebd., $\$ 666$

$13 \mathrm{Vgl}$. Zöllner (wie Anm. 1), 130-131 und Kat. Nr. 20-24. Vgl. hierzu auch Ernst $\mathrm{H}$. Gombrich, Ideal and Type in Italian Renaissance Painting, in: Ernst H. Gombrich, New Light on Old Masters, Oxford 1986, 89-124. 14 Leonardo da Vinci (wie Anm. 9), § 108. 
II.

"Jeder MALER MALT SiCh SELBST $«$. DiE THEORIE DER

\section{Automimesis}

Gegen die Verwendung immer derselben Figurentypen hat sich explizit nicht nur Leonardo gewandt, sondern auch einer seiner Zeitgenossen. So klagt zwischen 1497 und 1499 Gasparo Visconti, ein Hofdichter der Sforza, über einen namentlich nicht genannten Maler, der die wichtigste Voraussetzung für eine wirklichkeitsgetreue Darstellung ignoriert habe. Dieser Maler male zu den verschiedensten Gelegenheiten stets sich selbst, auch wenn er etwas ganz anderes darstellen wolle. Nicht allein sein schönes Antlitz bilde er ab, sondern sogar seine Gesten und Bewegungen. Auch fehle ihm für eine konzentrierte künstlerische Tätigkeit die notwendige Disziplin. ${ }^{15}$ Die von Visconti thematisierte Tendenz des Malers, sich unwillentlich selbst darzustellen, ist eine poetische Variante des bekannten toskanischen Sprichworts "Ogni pittore dipinge sé» (Jeder Maler malt sich selbst). ${ }^{16}$ Leonardo war zur Entstehungszeit des Sonetts der prominenteste Künstler der Toskana am Mailänder Hof, und er hatte ab circa 1492 wiederholt gegen die in diesem Sprichwort zum Ausdruck gebrachte Tendenz der Selbstdarstellung polemisiert. Es kann also kaum ein Zweifel daran bestehen, dass sich die Polemik Viscontis auf ihn bezog. Wie ernst es Leonardo mit seiner Ablehnung der von Visconti gegeißelten unwillentlichen Selbstdarstellung war, zeigen seine diesbezüglichen Formulierungen im Malereitraktat. Dazu ein Beispiel:

"Ein Maler, der plumpe Hände hat, wird ebensolche in seinen Werken machen, und dasselbe wird ihm bei jedem Körperteil passieren, wenn ein langes Studium ihn nicht davon abhält. So achte also, Maler, genau auf jenen Teil, der in deiner Person am hässlichsten ist und versuche dich mit deinem Studium davor $\mathrm{zu}$ schützen. Denn wärest du von bestialischer Gestalt, deine Figuren würden ebenso und geistlos aussehen, und ebenfalls wird sich jeder Teil, der gut oder traurig an dir ist, zum Teil in deinen Figuren zeigen. [...] Und wärest du hässlich, so würdest du unschöne Gesichter auswählen und hässliche Gesichter machen - wie viele Maler, denn oft ähneln die Figuren ihrem Meister «. ${ }^{17}$
15 Gasparo Visconti, I canzonieri per Beatrice d'Este e per Bianca Maria Sforza, hrsg. v. P. Bongrani, Mailand 1979, 117-118. 16 Zum Folgenden vgl. Martin Kemp, »Ogni dipintore dipinge sé«. A Neoplatonic Echo in Leonardo's Art Theory?, in: C. H. Clough (Hrsg.), Cultural Aspects of the Italian Renaissance. Essays in Honour of Paul Oskar Kristeller, New York 1976, 311-323; Frank Zöllner, >Ogni pittore dipinge sér. Leonardo on »automimesis«, in: $M$. Winner (Hrsg.), Der Künstler über sich in seinem Werk. Internationales Symposium der Bibliotheca Hertziana, Rom 1989, Weinheim 1992 , 137-160; Ders., Kunst und Wissenschaft: Leonardo zwischen »automimesis« und Proportionslehre, in: Denkströme, 4, 2009, 42-57. 17 Richter (wie Anm. 10), §̧ฐ 586-587. 
Die Tendenz der unwillentlichen Selbstdarstellung lässt sich allerdings mildern oder ganz vermeiden, wenn man ihre Ursa18 Leonardo da Vinci (wie Anm. 9), 5108. chen erkannt hat und ihnen entgegenwirkt. Hierzu schreibt Leonardo einige Jahre später: Er habe mehrfach über den Wiederholungs- und Selbstdarstellungszwang nachgedacht und dabei erkannt, dass dieser Defekt auf die Seele und ihr Urteilsvermögen zurückgehe. Die Seele nämlich forme den Körper nach einem gewissen Bilde, beispielsweise mit krummer oder dicker Nase, mit langen oder kurzen Gliedern, und dieselbe Seele wiederhole diesen Prozess des Bildens beim Malen, indem sie den Künstler immer nur krumme oder dicke, lange oder kurze Gliedmaßen malen lasse. Die Seele führe also die Hand des Malers ebenso, wie sie ihm zuvor seit der Geburt seine körperliche Gestalt bestimmt habe. ${ }^{18}$ Die Wesensverwandtschaft zwischen dem physiologisch bedingten Ausdruck in der Gestalt des eigenen Körpers einerseits und dem Schöpfungsprozess des Künstlers andererseits führt also zwangsläufig zu einer unwillkürlichen und ungewollten Selbstreproduktion des Künstlers in seinem Werk: Jeder Maler malt immer denselben Typus, der seiner eigenen, durch die Seele determinierten Gestalt ähnelt. Durch eine spezielle Schulung kann der Maler dieser unwillentlichen Selbstdarstellung allerdings wirksam entgegentreten, denn die Funktionen des Gehirns sind manipulierbar und zwar durch unermüdliches Üben, etwa durch das Zeichnen. Schult der Künstler beispielsweise seine Fähigkeit, Figuren zu zeichnen, dann nimmt das Gehirn das Erlernte in sich auf. Diese Schulung wiederum kann gezielt gegen die unbeabsichtigte künstlerische Reproduktion des Selbst eingesetzt werden, indem eben jene Figuren erlernt werden, die dem eigenen Wiederholungstrieb entgegenstehen. Ein dicker Maler müsste also dünne Figuren zeichnen und umgekehrt, oder am besten natürlich perfekte Figuren oder auch Musterfiguren. Konkret beschreibt Leonardo dieses Vorgehen folgendermaßen:

"Der Maler soll sich seine (Muster-) Figur nach der Regel eines natürlichen Körpers bilden, der in der Proportion allgemein für lobenswert gilt. Außerdem soll er sich selbst ausmessen und feststellen, in welchem Teil er sehr viel oder wenig von jener vorgenannten 
lobenswerten Figur abweicht. Und wenn er das gelernt hat, dann muss er mit seinem ganzen Studium dafür sorgen, dass er nicht bei den von ihm geschaffenen Figuren in die gleichen Mängel verfällt, die sich an seiner eigenen Person finden. ${ }^{19}$

Doch nicht nur das genaue Studium und der Gebrauch von Musterfiguren helfen im Kampf gegen die unwillentliche Selbstdarstellung, die Automimesis, und den überzogenen Gebrauch stereotyper Figuren. Neben der aufwändigen Schulung des Geistes nennt Leonardo noch eine weniger anstrengende Möglichkeit: Zur Manipulation des eigenen Urteils solle der Künstler seinen Geist in kurzweiligen Spaziergängen den Mannigfaltigkeiten der Natur aussetzen. Der Geist wende sich so den verschiedenen Objekten zu und könne auf diesem Wege ein gewisses Reservoir guter und weniger guter Sachen in sich aufnehmen. ${ }^{20}$ Selbst für Leonardos scherzhaft anmutende Empfehlung, den Geist gelegentlich von zu eifrigem Studium zu entlasten, existiert Bestätigung von unabhängiger Seite. So mag man Gaspare Viscontis nicht ganz ernst gemeinten Vorwurf der Disziplinlosigkeit (s.o.) auf diese Empfehlung Leonardos beziehen. Zudem schildert der 1485 geborene Matteo Bandello, der bis 1501 im Kloster von Santa Maria delle Grazie weilte und als Augenzeuge von Leonardos Vorgehensweise gelten kann, tatsächlich einen eher entspannten als disziplinierten Arbeitsstil des Künstlers. In der Vorrede zur 58. Erzählung seiner zuerst 1554 erschienenen Novellensammlung schreibt er, Leonardo habe seine Arbeiten am »Abendmahl« oft mehrere Tage unterbrochen, dann aber auch Stunden in abwägender Kontemplation vor dem Bild verbracht. Andererseits sei er oft spontan, ganz nach Lust und Laune, zu dem Gemälde geeilt, um den einen oder anderen Pinselstrich auszuführen und dann seine Arbeitsstätte gleich wieder zu verlassen. ${ }^{21}$ Auch hier bestätigen also zeitgenössische Quellen Leonardos Überlegungen zu seiner Arbeitsweise.

III.

\section{DER KONTEXT DES "ABENDMAHLS «}

Die Rezipienten des Bildes haben fast ausschließlich die künstlerische Leistung Leonardos in der meisterhaften Darstellung
19 Ebd., § 109.

20 Ebd., $\$ 56$.

21 Villata (wie Anm. 5)

§346. Eine deutsche

Übertragung findet sich bei Lüdecke (wie Anm. 8), 11-12. 


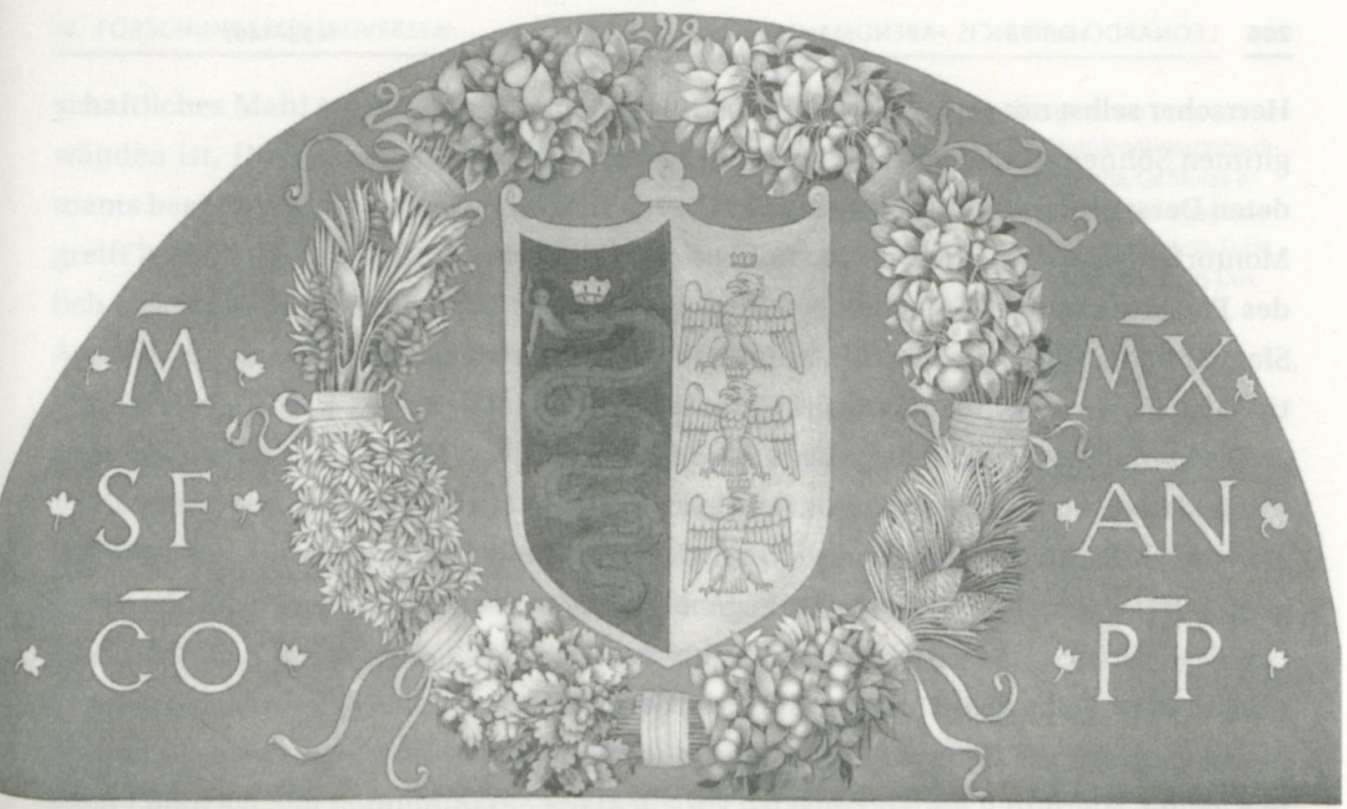

ABB. 5: LEONARDO DA VINCI, ABENDMAHL MIT WAPPENFELD, CA. 1495-1497, MAILAND, SANTA MARIA DELLE GRAZIE, NORDWAND DES REFEKTORIUMS, REKONSTRUKTION BRAMBILLA BARCILON/MARANI, 1990

zum "Abendmahl« auf der Nordwand her, da dort das gemeinsame Mahl selbst dargestellt ist.

Weitere Bezüge existieren zwischen dem »Abendmahl« und den darüber angebrachten Lünettenbildern (Abb. 2 u. 5). Diese, etwa zeitgleich entstandenen Bilder waren im 18. Jahrhundert übertüncht und erst 1853/1854 wieder sichtbar gemacht worden, blieben aber noch bis zur jüngsten Restaurierung 1999 fast unlesbar. Daher fanden sie in der Fachliteratur kaum Beachtung. Sie sind allerdings von kaum zu überschätzender Bedeutung für das Verständnis der Gesamtausstattung des Refektoriums und damit des »Abendmahls«. Die mittlere Lünette zeigt das gemeinsame Wappen Ludovico Sforzas und seiner Frau Beatrice d'Estes. Links daneben (also heraldisch gesehen rechts) findet sich das Wappen des ältesten Sforza-Sohnes Massimiliano; der Wappenschild auf der rechten Seite verweist mit der noch erkennbaren Inschrift auf das Herzogtum Bari, das Ludovicos zweiter Sohn Francesco 1497 erhielt. Unmittelbar über dem »Abendmahl« finden sich also gleichsam heraldische Porträts der Stifterfamilie: der Mailänder 
Herrscher selbst mit seiner Gattin flankiert von seinen beiden legitimen Söhnen. Und auch auf der Südwand mit der 1495 vollendeten Darstellung einer Kreuzigung Christi von Giovanni Donato Montorfano sind die Sforza präsent, denn an den beiden Seiten des Freskos knien in Gestalt von Stifterporträts links Ludovico Sforza mit dem ältesten Sohn Massimiliano und rechts seine Gattin Beatrice mit dem jüngeren Sohn Francesco.

Die auffällige Präsenz der Sforza im Refektorium von Santa Maria delle Grazie ergibt sich aus der Geschichte des nach 1460 errichteten Klosterkomplexes. Schon ab 1492 ließ Ludovico den gerade fertig gestellten Chor der dazugehörigen Kirche zu einer Grablege für sich und seine Familie umbauen. Der Chorbereich und weitere Teile des Kirchenraums wurden damit zu einem Mausoleum für die Sforza und für die mit ihnen politisch liierte Klientel. Hierdurch erhielt zunächst vor allem der Sakralraum als Grablege der Herrscherfamilie eine dynastische Komponente, die teilweise auch in der Gesamtausstattung des Refektoriums zum Ausdruck gelangt. So spiegeln die Wappen in den Lünetten oberhalb des »Abendmahls" mit ihren Inschriften unmittelbar den damals aktuellen Stand der Machtpolitik und der Bemühungen Ludovicos um dynastische Selbstvergewisserung wider. Er selbst wird in der mittleren Lünette in seiner offiziell erst ab 1495 anerkannten Würde als DVX MEDIOLANI (Herzog von Mailand) genannt. Die Wappen links und rechts davon verweisen auf die Söhne Ludovicos und damit auf den Fortbestand der noch jungen Dynastie. Schließlich finden sich in den unmittelbar angrenzenden Lünetten der beiden Langwände noch zwei leere Wappenfelder, die den Eindruck erwecken, als ob weitere Nachkommen Ludovicos hier mit ihren heraldischen Zeichen hätten dargestellt werden sollen. Mit diesem Arrangement zielte Ludovico auf eine Anbindung seiner familiären Geschicke an das sakrale Bildgeschehen und den räumlichen Kontext. Tatsächlich befindet sich die in den Wappen der Lünetten und in den Stifterporträts präsente Familie in der Gemeinschaft jener Heiligen und Seligen des Dominikanerordens, die im Ornamentband der Langwände porträtiert sind. Die Sforza-Familie wird gleichsam zu einem imaginären Teil jener »Familie« der Dominikaner, deren gemein- 
schaftliches Mahl auch das Thema der Inschriften auf den Langwänden ist. Die genealogische Struktur des gesamten Arrangements beeinflusst sogar die Darstellung des »Abendmahls", ja sie greift letztlich direkt in Leonardos Bildgestalt ein. Denn tatsächlich korrespondiert die Einteilung der in Gruppen angeordneten Apostel, die bekanntlich als revolutionäre Neuerung Leonardos gilt, mit dem Rhythmus der Lünetten: Die Jünger an den beiden äußeren Enden des Tisches befinden sich jeweils unterhalb der beiden kleineren Bögen, die beiden inneren Gruppen zusammen mit Christus unter dem mittleren Bogen. Hierbei fällt Judas aus der beschriebenen Gruppenbildung heraus, denn allein sein Kopf lässt sich nicht genau einer der Lünetten zuordnen. Der Verräter sitzt bezeichnenderweise außerhalb der ordnenden Struktur. Leonardos Gemälde und sein Kontext waren also eng mit den Versuchen Ludovico Sforzas verknüpft, seinen dynastisch motivierten Versuchen der Selbstdarstellung Ausdruck zu verleihen. ${ }^{23}$ Diese Möglichkeit einer Kontextualisierung hat allerdings in der Forschung kaum Interesse geweckt. Andere Fragen standen häufiger im Mittelpunkt; zwei davon seien abschließend erörtert.

\section{IV.}

\section{FORSCHUNGSKONTROVERSEN: DARSTELLUNG UND}

\section{PERSPEKTIVISCHE KonSTRUKTION}

Leonardos "Abendmahl « unterscheidet sich nicht allein durch seine Ausdrucksvielfalt von anderen zeitgenössischen Beispielen, es stellt auch in anderer Hinsicht eine Ausnahme dar. So gehört das Gemälde zu den wenigen zwischen 1430 und 1499 in der Lombardei entstandenen Gemälden dieser Art, die sich in einem Refektorium befinden und dabei eindeutig die Verratsankündigung bzw. den Moment unmittelbar danach darstellen. Hieraus ergibt sich in methodischer Hinsicht die Frage nach dem Darstellungstyp, wobei vor allem zwei Tendenzen zu beobachten sind: Der größere Teil der Forschung sieht abgesehen von der Verratsankündigung vor allem die Einsetzung des Sakraments der Eucharistie dargestellt, denn Jesus verweise mit beiden Händen auf Brot und Wein vor ihm auf dem Tisch und damit auf eucharistische Symbole, die für den Leib und das Blut Christi stehen. ${ }^{24}$ Ausgehend von den ent- 
sprechenden Überlegungen Leo Steinbergs gehen weitergehende Deutungen dahin, die Ikonographie zudem vor dem Hintergrund des dominikanischen Engagements für die Transsubstantiationslehre zu verstehen. Leonardos Darstellung verweise demnach auf die wahre Präsenz des Leibes und des Blutes Christi während der Messe, die mit der Einsetzung des Sakraments der Eucharistie während des Abendmahls vorweg genommen werde. Hierbei habe auch der 1495 zum Prior des Klosters ernannte Vincenzo Bandello (der Onkel Matteo Bandellos, s.o.) eine Rolle gespielt und zur Ikonographie des Gemäldes beigetragen. ${ }^{25}$

Wesentlich einfacher und im Grunde auch schlüssiger ist die Ansicht Creighton Gilberts, der nur die Verratsankündigung dargestellt sieht und seine Argumentation vor allem mit gattungsgeschichtlichen Überlegungen begründet. Tatsächlich machen fast alle Abendmahlsdarstellungen der zweiten Hälfte des 15. Jahrhunderts eine eucharistische Bedeutung durch symbolische Gegenstände kenntlich. So verweisen oft ein Kelch und gelegentlich auch eine Hostie eindeutig auf das Sakrament der Eucharistie. ${ }^{26}$ Diese Eindeutigkeit fehlt in Leonardos Gemälde. Zudem finden sich vorwiegend eucharistisch gemeinte Abendmahlsdarstellungen kaum in Refektorien, sondern in erster Linie auf Altarbildern (etwa in Predellentafeln) und somit in Werken, die für einen liturgisch zweifellos mit der Eucharistie verbundenen Kontext bestimmt waren und wo eucharistische Anspielungen auch einen Sinn machten. Hingegen assoziiert man mit der Verratsankündigung nicht unbedingt die Eucharistie.

Vergleichsweise große Aufmerksamkeit hat zudem die Perspektivkonstruktion von Leonardos »Abendmahl«gefunden, gilt doch ein nach Gesetzen der Zentralperspektive konstruierter Bildraum als herausragende Eigenschaft der Renaissancemalerei. Wohl nicht zuletzt aufgrund seiner suggestiven Perspektive ist Leonardos Bild bis heute die bekannteste Variante dieses Themas. Wie schon die Florentiner Künstler vor ihm stellt Leonardo das letzte Mahl des Herrn in einem nach Regeln der Zentralperspektive konstruierten bühnenartigen Raum dar. Den wirklichen oder vermeintlichen Konstruktions- und Berechnungsprinzipien von Leonardos Perspektive wurden zahlreiche, einander wi-
25 Marco Rossi, Alessandro Rovetta, II Cenacolo di Leonardo. Cultura domenicana, iconografia eucaristica e tradizione lombarda, Mailand 1988, 58-66; Dominique Rigaux, A la table du Seigneur. L'Eucharistie chez les primitifs italiens (1250-1497) Paris 1989.

26 Creighton Gilbert, Last Suppers and their Refectories, in: Charles Trinkaus, Heiko A. Obermann (Hrsg.), The Pursuit of Holiness in Late Medieval and Renaissance Religion, Leyden 1974 , 371-407. 
dersprechende Studien gewidmet. ${ }^{27}$ Deren Maßzahlen und Konstruktionen halten jedoch einer kritischen Überprüfung nicht stand. ${ }^{28}$ Zudem bleibt bislang völlig unklar, wie und warum Leonardo beispielsweise ein komplexes Proportionssystem auf die Nordwand hätte bringen sollen. Unstrittig ist nur, dass der ideale Betrachterstandpunkt der Perspektive rund vier Meter über dem Fußbodenniveau und somit weit über der normalen Augenhöhe des Betrachters liegt. Dabei vermied Leonardo eine stark untersichtig angelegte Darstellung, wie sie in Mailand im Umkreis Donato Bramantes und Vincenzo Foppas bis dahin populär war. ${ }^{29}$

Ein wichtiger Aspekt von Leonardos Darstellungsmodus ist zweifellos der Fluchtpunkt. Tatsächlich laufen die Perspektivlinien in der rechten Schläfe des Hauptes Christi zusammen, womit die zentrale Stellung des Erlösers im Bildgeschehen auch formal eine Betonung erfährt. Die Perspektive wird also, was auch für einige andere Gemälde des 15. Jahrhunderts gilt, zum Bedeutungsträger. ${ }^{30}$ Ebenso konventionell ist die Platzierung des Abendmahls auf der Schmalwand eines Refektoriums, die Leonardo vor allem aus seiner Heimatstadt Florenz kannte. Hierbei fingiert die Perspektivkonstruktion die Fortsetzung des Betrachterraumes im Bildraum, der sich wie eine Bühne weit über den Köpfen der Betrachter zu öffnen scheint. Auf einer im wahrsten Sinne des Wortes übergeordneten Bildraumebene fungiert die sakrale, ins Bild gesetzte Handlung als Exemplum für die im Realraum speisenden Mönche, was durch die entsprechenden Inschriften mit ihren Verweisen auf das gemeinschaftliche Mahl eigens betont wird. Der Bildraum setzt also den Realraum fort, dies aber auf einer höheren Ebene. Die Mönche speisen in imaginärer Gemeinschaft mit Christus und seinen Jüngern, aber eben nur imaginär.

In jedem Fall vermittelt also die Perspektivkonstruktion zwischen Realraum und Bildraum. Das Thema der Perspektive ist damit eines der Öffnung und Verbindung von Räumen, ebenso eines der Verbindung der Darstellung des sakralen Geschehens zum realen zeitgenössischen Kontext. Diese kontextuelle Ver- und Einbindung hat bezeichnenderweise wenig zur Kanonisierung des "Abendmahls« beigetragen. Eher das Gegenteil ist der Fall, denn ein Blick auf die Rezeptions- und Forschungsgeschichte des Ge-
$27 \mathrm{Vgl}$. als aktuelles

Beispiel: Matthew Landrus, Leonardo's "Last Supper«, in:

Raccolta Vinciana 32, 2007, 43-100; zusammenfassend Zöllner (wie Anm. 1), 231;

Steinberg (wie Anm.1), 153-194.

$28 \mathrm{Kim} \mathrm{H}$. Veltman, Studies on Leonardo da Vinci I. Linear Perspective and the Visual Dimensions of Science and Art, München 1986, 28-29; James Elkins, The Case Against Surface Geometry, in: Art History, 14. 1991, 143-174.

29 Giulio Bora, Prospettiva lineare e prospettiva »Des Perdimentik. Un dibattito sullo scorcio del Quattrocento, in: Paragone, 50 (595), 1999, 3-45.

30 Alexander Perrig,

Masaccios »Trinità und der Sinn der Zentralperspektive, in: Marburger Jahrbuch für Kunstwissenschaft, 21, 1986, 11-43, bes. 26-27; Frank Büttner, Perspektive als rhetorische Form. Kommunikative Funktionen der Perspektive in der Renaissance, in: Joachim Knape (Hrsg.), Bildrhetorik, Baden-Baden 2007, 201-231. 
mäldes lehrt, dass der ursprüngliche Kontext im Gegensatz zur Perspektive in der Wahrnehmung durch den Betrachter und in der wissenschaftlichen Würdigung des »Abendmahls " gar keine oder nur eine untergeordnete Rolle gespielt hat. Kanonisierung und Kontextualisierung eines Kunstwerks scheinen also eher in gegensätzliche Richtungen zu laufen. 


\section{GRUNDLEGENDE LITERATUR ZUM THEMA:}

Pinin Brambilla Barcilon, Pietro C. Marani, Leonardo.

L'ultima cena, Mailand 1999

Creighton Gilbert, Last Suppers and their Refectories, in: Charles Trinkaus, Heiko A. Obermann (Hrsg.), The Pursuit of Holiness in Late Medieval and Renaissance Religion, Leyden 1974, 371-407

Ludwig H. Heydenreich, Invito a Leonardo. L'ultima Cena, Mailand 1982 (zuerst engl, 1974)

Emil Möller, Das Abendmahl des Lionardo da Vinci, BadenBaden 1952

Alessandro Nova, Sabine Feser (Hrsg.), Giorgio Vasari, Das Leben des Leonardo da Vinci, übers. v. Victoria Lorini, Berlin $2006,28-32,81-84$
Dominique Rigaux, A la table du Seigneur. L'Eucharistie chez les primitifs italiens (1250-1497), Paris 1989

Marco Rossi, Alessandro Rovetta, II Cenacolo di Leonardo. Cultura domenicana, iconografia eucaristica e tradizione lombarda, Mailand 1988

Leo Steinberg, Leonardo's Incessant Last Supper, New York 2001

Kim H. Veltman, Studies on Leonardo da Vinci I. Linear Perspective and the Visual Dimensions of Science and Art, München 1986

Frank Zöllner, Leonardo da Vinci. Sämtliche Gemälde und Zeichnungen, 3. Aufl., 2 Bde., Köln u.a. 2011, Bd. 1, 122-139, 206-207, 230-232

\section{BILDNACHWEIS:}

ABB. 1: ZOLLNER, 2011. ABB. 2: MOLLER, 1952. ABB. 3: ARCHIV DES AUTORS. ABB. 4: MARTELLI, 1980 ABB. 5: BRAMBILLA BARCILON/ MARANI, 1990. 